
\title{
3 Research Square \\ The Difference of Antibiotic Resistance Genes in Gut Microbiota Among Yak, Beef and Dairy Cattle by Metagenomic Approach
}

\section{Weiwei Wang}

Chinese Academy of Agricultural Sciences Lanzhou Institute of Husbandry and Pharmaceutical Sciences

\section{Xiaojuan Wei}

Chinese Academy of Agricultural Sciences Lanzhou Institute of Husbandry and Pharmaceutical

Sciences

\section{Lingyu Wu}

Chinese Academy of Agricultural Sciences Lanzhou Institute of Husbandry and Pharmaceutical Sciences

\section{Xiaofei Shang}

Chinese Academy of Agricultural Sciences Lanzhou Institute of Husbandry and Pharmaceutical

Sciences

\section{Fusheng Cheng}

Chinese Academy of Agricultural Sciences Lanzhou Institute of Husbandry and Pharmaceutical Sciences

\section{Bing Li}

Chinese Academy of Agricultural Sciences Lanzhou Institute of Husbandry and Pharmaceutical

Sciences

\section{Xuzheng Zhou}

Chinese Academy of Agricultural Sciences Lanzhou Institute of Husbandry and Pharmaceutical Sciences

Jiyu Zhang ( $\sim$ zhangjiyu1967@163.com )

Chinese Academy of Agricultural Sciences

\section{Research}

Keywords: antibiotic resistance genes, metagenomic approach, selective pressure, feeding patterns, mobile genetic elements

Posted Date: September 2nd, 2020

DOl: https://doi.org/10.21203/rs.3.rs-63086/v1 
License: (c) (i) This work is licensed under a Creative Commons Attribution 4.0 International License. Read Full License 


\section{Abstract}

The drug resistance has been partly driven by the overuse of antimicrobials in agricultural feeding. Better understanding of the antibiotic resistance in gut of bovine is needed to assess its potential effects based on metagenomic approach and analysis. In this study, we collected 40 fecal samples to explore the drug resistance deriving from antibiotics in bacterial community by an analysis of the diversity and difference of antibiotic resistant genes (ARGs) in gut microbiota from yak, beef and dairy cattle. 1688 genes were annotated, including 734 ARG subtypes in summary. The ARGs were related with tetracycline, quinolone, $\beta$-lactam, aminoglycoside, in accordance with the antibiotics widely used in clinic for humans or animals. The emergence, prevalence and differences in resistance genes in the intestines of yaks, beef and dairy cattle may be caused by the selective pressure of different feeding patterns. Additionally, the abundance of ARGs in yak was lower than in beef and dairy cattle, while the abundance of integron, a kind of mobile genetic elements (MGEs), was higher than those in beef and dairy cattle. Furthermore, the results of this study demonstrated that a comprehensive profile of various ARGs among yak, beef and dairy cattle for providing a basic research.

\section{Introduction}

Diverse and abundant of antibiotics are used to control bacterial diseases and promote the growth of livestock, which may lead to antibiotic resistant bacteria (ARBs) that are widespread in the world (1). The antibiotic use as feeding additives to promote the growth in livestock farming has become a serious problem, which leads to the result that antibiotic resistance is getting worse. The researchers have also showed that the antibiotic consumption in animals are already used twice as much as that in humans(2). According to FDA, about $80 \%$ antimicrobials are used for animals in the USA(3). Statistically, American uses 14600 tons of antimicrobials and China uses 97000 tons of antimicrobials for animals in 2012(3) (4). Moreover, parent compounds and metabolites from antibiotics were detected in excretion of animals because of incomplete metabolization and poor absorption in the gastrointestinal tract(5). ARBs harboring ARGs can be delivered into the environment via animal feces. Feces directly or indirectly lead to the wide spread of ARGs in the environment, and there is a risk of eventual transmission to humans. And animal fecal bacteria community was a vast reservoir of ARGs deriving from commensals and pathogens of humans(6). Studies on the diversity and high abundance of drug-resistant genes in animal intestinal bacterial community show that it will be very difficult for human to prevent and control animal bacterial diseases if the ARGs are transferred and prevalent in pathogens. It is very important to study the resistance of gut microbiome in animals for the effective prevention and control of bacterial diseases, the establishment of strategies to prevent the transfer of drug resistance in bacteria and the guidance of clinical drug use. At the same time, it is also of great significance for the public health and food safety.

China's breeding industry has developed rapidly and was the world's largest consumer of antibiotics for animals in the year of 2010(7). Enclosed and intensive production which embodied the characteristics and advantages of modern breeding husbandry is a mostly effective way for farming. However, with the growth of feeding density, the infectious disease can quickly spread among animals(8). Therefore, the 
control of bovine bacteria disease and the research of antibiotic drug resistance are very important. To reduce the risk of the spread of ARGs, researchers have devoted considerable effort to studying antibiotic resistance in carnivorous animals like bovine. Some researchers have even claimed that the different level of antibiotics resistance can also be found in the different animal cohorts(9).

It's shown that ARGs can be transferred horizontally in human and animals' intestines, soils, sediments, waters (10)(11)(12)(13). And the transfer rate of HGT in intestinal tract is 25 times higher than that in environments(14). Multiple MGEs carrying ARGs were isolated from clinical ARBs. Through the way of horizontal gene transfer (HTG), MGEs is an important element to drive the ARG dissemination. The frequency of transmission of HTG is much higher than of other genes(15)(16). However, the clear mechanism of ARGs transmission driven by the HGT was still little known in bovine.

A growing number of researchers focused on detecting the microbial diversity using the metagenomic approaches in the gastrointestinal tracts of various animals(17). And the rare and wild taxa in the microbiota of animal feces can be explored through metagenomic approach(16). The purpose of this study is to achieve comprehensive profile in 40 bovine fecal samples harboring the ARGs and MGEs and to evaluate the difference and diversity of ARGs and MGEs among different species (yak, beef and dairy cattle) based on metagenomic approach.

\section{Materials And Methods}

\subsection{Sampling}

40 fecal samples were collected from different breeding pattern and areas in China, including Xinjiang Province, Gansu Province, Qinghai Province and Sichuan Province. Bovine was maintained on the same diet for 28 days prior to the experiment to decrease the variation. We randomly selected the healthy bovine in 4 provinces, and fresh fecal samples were collected quickly and then transported into liquid nitrogen. The fecal samples then were placed in $-80^{\circ} \mathrm{C}$ until DNA extraction. The basic information summary of 40 fecal samples was shown in Table S1.

\subsection{DNA extraction and metagenome sequencing}

DNA was extracted from fecal samples using the method of CTAB following the instruction. DNA degradation degree and potential contamination was monitored on $1 \%$ agarose gels. And DNA concentration was measured using Qubit ${ }^{\circledR}$ dsDNA Assay Kit in Qubit ${ }^{\circledR}$ 2.0 Fluorometer (Life Technologies, CA, USA) and purity was measured using NanoPhotometer ${ }^{\circledR}$ spectrophotometer (IMPLEN, CA, USA). A total amount of $1 \mu \mathrm{g}$ DNA per sample was used for shotgun library construction. Metagenomic sequencing was performed using Illumina Hiseq $X$ ten platform with the sequencing strategy of Index 150 PE (paired-end sequencing). The specific processing steps are as follows: a) remove the reads which contain low quality bases; $b$ ) remove the reads in which the $\mathrm{N}$ base has reached a certain percentage; $\mathrm{c}$ ) remove reads which shared the overlap above a certain portion with Adapter. Over 12Gb clean reads were 
detected via metagenomic approach in each sample and the proportion of high-quality reads among all raw reads from each sample was no less $95 \%$.

\subsection{Metagenome assembly and ORF prediction}

After treatment, the high-quality clean data were assembled using SOAPdenovo2 assembly software(18). All samples' clean data are compared to Scaftigs by Soap Aligner(19). Based on the Scaftigs of single sample and mixed assembly, gene catalogue was constructed to predict gene(19)(20)(21)(22).

The open reading frame (ORF) within Scaftigs ( $\geq 500 \mathrm{bp}$ ) was MetaGeneMark. All of the ORF should be filtered using CD-HIT with a minimum similarity of identity $95 \%$, coverage $90 \%$ and be selected by the longest gene sequence as the standard for achieving the non-redundant Unigenes(23)(24).

\subsection{Relative abundance analysis}

Based on the number of mapped reads and the length of gene, the abundance information of each gene in each sample has been calculated(25). For each gene, "r" referred to the number of read pairs; "L" referred to the length of the corresponding gene; "G" referred to the relative abundance. The relative abundance of gene was calculated by the following formula:

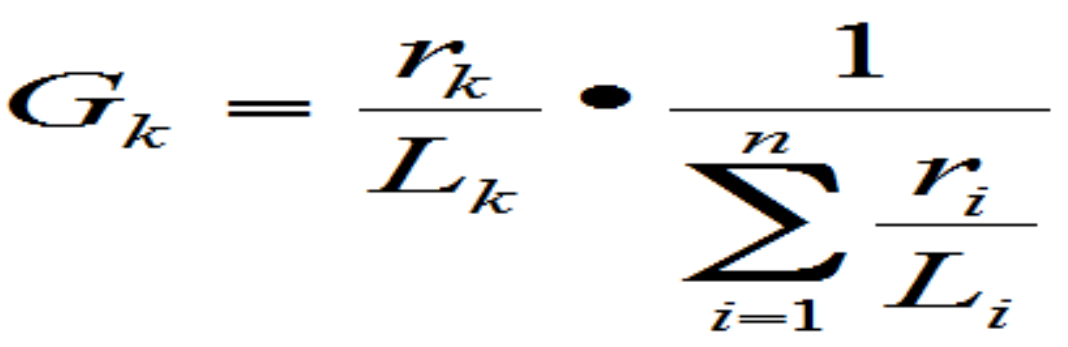

\subsection{Taxonomy prediction}

Unigenes sequences from each sample were then compared against NR database of NCBI(Version: 201801-02) by using DIAMOND with the parameters (blastp囚evalue $\leq 1 e-5)(26)$. Subsequently, we adopted the LCA algorithm(LCA parameters: mini-score 35, top percentage 10\%) which is applied in systematic classification of MEGAN software to make sure the species annotation information of sequence(27).

\subsection{Identification of ARGs}

No-redundant gene sets were compared with CARD database(https://card.mcmaster.ca/) using Resistance Gene Identifier (RGI) software to annotate antibiotic resistant genes(blastp, evalue $\leq 1 \mathrm{e}-30$ ) (28)(29)(30). According to the results of gene annotation to analyze the species corresponding to the ARG.

\section{Results}




\subsection{Identification and taxonomy of ARGs}

The metagenomic library was constructed for evaluating ARGs reservoir in gut of yak, beef and dairy cattle. 40 fecal samples were tested using Illumina platform and obtained $548.3662 \mathrm{Gbp}$ of high-quality and average on each sample was $13.0564 \mathrm{Gbp}$. And the detailed data summary was exhibited in Table S2.

According to the annotation results compared with CARD, the species information corresponding to the drug-resistant genes can be analyzed and the dominant flora carrying the drug-resistant genes can be reflected. In comparison of the distribution of the bacterial gene sets and ARGs at phylum level, Firmicutes, Bacteroidetes and Proteobacteria were dominant, accounting for $53 \%$ to $33 \%, 15 \%$ to $11 \%$ and $5 \%$ to $9 \%$ in the total amount of bacteria of yak, respectively (Fig.1). For beef cattle, Firmicutes, Bacteroidetes were dominant, accounting for $39 \%$ to $32 \%$ and $34 \%$ to $15 \%$ in the total amount of bacteria. And for dairy cattle, Firmicutes, Bacteroidetes were dominant, accounting for $34 \%$ to $28 \%$ and $41 \%$ to $16 \%$ in the total amount of bacteria. These asymmetric relationships suggest that Firmicutes are more likely to carry resistant genes in bovine fecal samples.

\subsection{Diversity and distribution of ARGs in gut}

Approximately $85-99 \%$ of microbes cannot be cultivated in the laboratory, which limits our understanding of microbes, including those with ARGs(31). Therefore, the metagenomic approach was used to investigate the distribution and diversity of drug resistance genes in the intestinal tract of bovine. A total of 5701,582 predictive genes were annotated after the original redundancy, and 1688 genes could be annotated by CARD database, including 734 ARG types. To eliminate the differences in the number of ARGs caused by the differences in sample data, the number of ARGs annotated to each Gb of data in different groups was calculated. To be specific, the number of ARG/Gb in yak group(17.83 \pm 2.67$)$ was much lower than in group beef $(18.28 \pm 2.56)$ and dairy cattle $(19.25 \pm 1.77)(p<0.001)$. And the detailed information of ARGs in each sample was shown in Table S3 (a\&b\&c).

According to the abundance information of ARG in each sample, the top 30 ARGs are used to draw the heatmap (Fig.2). And it's seen that heat map hierarchical clustering based on the relative abundance of each ARGs showed that yak samples were clustered individually and weren't clustered with beef and dairy cattle samples.

\subsection{The relative abundance of ARGs in gut}

There was some difference of ARGs number in gut among yak, beef and dairy cattle. Specially, the number of ARGs in yak group was lower than in other groups (Fig.3). Additionally, the relative abundance of ARGs was significantly higher in dairy cattle and beef than that in yak $(p<0.001)$. And the detailed relative abundance of ARGs in each sample was shown in Table S4.

Starting from the relative abundance table of resistance genes, the ARGs content and percentage in each sample were calculated, and the ARGs results of the maximum abundance ranking top 20 were screened 
as diagram in Fig.4a\&b. It's seen that there was a difference on the relative abundance of ARGs among yak group and other groups. Specifically, the yak of the relative abundance and relative percentage of the yak were different from those of beef and dairy cattle.

Fig.4 (a) represents the relative abundance of ARGs in each sample, and the unit PPM is the result of amplifying the original relative abundance data by 106 times; (b) represents the relative abundance of top20 ARGs in all ARGs, and others represent the total relative abundance of non-top 20 ARGs.

Fig.5 The top 30 ARG abundance clustering heat map. The right vertical axis is the ARG name, the left vertical axis is the ARG clustering tree, and the corresponding value of the intermediate heat map is the $Z$ value of ARG relative abundance in each row after standardized processing.

It showed that yak samples were clustered individually and weren't clustered with beef and dairy cattle samples in the Fig.5\&6. Interestingly, the abundance of tetracycline resistance genes (tetX, tetQ, tet44, tet 40 , tetO, tetW, tetW/N/W) were much higher in beef and dairy cattle than in yak. And the abundance of aminoglycoside resistance genes (VanRI, VanYA, VanRA, DHA-19), $\beta$-lactam resistance genes (OXA-363, PER-7) and multidrug resistance genes (abcA, AcrS) were much higher in yak than in beef and dairy cattle.

Fig.6 Heatmap variations of the relative abundance level of each top30 ARGs subtype among the group of yak, beef and dairy cattle. The right vertical axis is the name of ARG subtype, and the bottom of horizontal axis is the group name. The left vertical axis is cluster tree.

\subsection{Shared ARGs among yak faces, beef faces and dairy cattle faces}

To detect the distribution of shared ARGs among yak, beef and dairy cattle, the Venn diagram and Ternary plot were constructed. A sum of 318 ARGs were shared by faeces from yak, beef and dairy cattle (Fig.7). And the differences in the abundance of 318 ARGs for different drugs in three groups were also analyzed in ternary plot (Fig.8). The percentage of each certain ARGs in each group of gut is equal to its corresponding abundance which are divided by the sum abundance of ARGs in three groups of gut. Among these shared ARGs, the abundance of tetracycline genes, quinolone genes, $\beta$-lactam genes and macrolide genes were much higher in beef and dairy cattle than those in yak.

Fig.7 The Venn diagram showing the number of shared ARGs in yak gut, beef gut and dairy cattle gut

Fig.8 Ternary plot showing the abundance comparison of 318 shared ARGs in yak gut, beef gut and dairy cattle gut. The sum of the abundance for one species ARG in these three types of gut was set as $100 \%$. The percentage of each certain ARG in each gut is equal to its corresponding abundance which is divided by the abundance sum of this ARG in the three groups of gut.

Fig.9 The relative abundance of shared ARGs types assigned to each major antibiotic class among the group of yak, beef and dairy cattle. 
Moreover, the results showed that two antibiotic classes resistance genes consisting tetracyclines and $\beta$ lactams accounted for $>50 \%$ of the total ARGs in beef and dairy cattle (Fig.5). While, the results also showed that multidrug accounted for nearly $50 \%$ in yak (Fig.5).

\subsection{Occurrence and Abundance of MGEs}

By comparing with the IS finder database, a total of 153,981 MGEs were annotated, and the differences existed among three groups. Observational studies of mobile transfer elements showed that the abundance of integron in yak group was much higher in beef and dairy cattle (Pख0.0001) (Fig.10). But it is shown that there was no obvious difference between beef cattle and dairy cattle (P凶0.05). Moreover, the top ten most abundant integron varied from yak to beef and dairy cattle, the AP011957 were the most abundant type respectively in three groups (Fig.10b).

Fig.10 (a)The difference of relative abundance of mobile genetic elements (integron) among yak, beef and dairy cattle is shown in the sigbox. (**represents $p<0.005$, $* \star \star$ represents $p<0.001$, $* \star \star \star$ represents $p<0.0001)(b) T h e$ difference of the abundance of top ten mobile genetic elements (integron) in the yak, beef and dairy cattle.

\section{Discussion}

The main aim of this study was to analyze fecal resistome and bacterial community and to explore the effects of antibiotic selection pressure in yak, beef and dairy cattle using the comparative metagenomic approaches. Notably, fecal samples from bovine were collected by the same protocols and sequence platforms, the differences may exist because of different species. Moreover, under that condition, batch effects may exist because of samples from multi-site. In this study, we presume that differential selection pressure of antibiotics may contribute to the difference in drug resistance in gut microbiota of yak, beef and dairy cattle.

With the rapid fall in the cost of production and use of antibiotic, more and more subtherapeutic dose of antibiotics promoting the growth and preventing the disease are being used in the breeding industry(32) (33). The factor partly explains why there are plenty ARGs which are detected in food animals. High population density produces high demands for animal production, which leads to the production of highdensity feeding patterns. It's reported that a close relationship in the use levels of antibiotics in animals at a population level(34). And this high-density feeding patterns potentially affects the increase in selecting pressure on bacteria to become resistant(7). Generally, yak, beef and dairy cattle are raised in different feeding patterns. As is known, places at high altitudes of western China are home to yaks, where has $90 \%$ population of wild yak in the world(35). As a special species of China, comparing with beef and dairy cattle, the yak was seldom exposed to antibiotics and was farmed in low-density feeding pattern. To be specific, the yak lives in the low-density feeding pattern and the beef and dairy cattle live in the highdensity feeding pattern. Animals in the high-density feeding pattern have a greater risk of infection leading to antibiotic use than animals in the low-density feeding pattern. And high density feeding pattern even lead to zoonotic transmission and also acts as a huge reservoir of ARGs(36)(37)(38). Thus, in other 
words, beef and dairy cattle exposed to more antibiotics may be more likely to develop resistance than yaks. Therefore, the difference of abundance and diversity of ARGs among three groups may associated with antimicrobial selection pressure caused by different feeding patterns. The data in this study showed that the difference between yak and other two groups relating on predominant ARGs types (top100) was striking, and the abundance and diversity of ARGs in yak was lower than in other groups. It partly suggested that the ARGs in the gut of yak, beef and dairy cattle are due to selection pressure. Many studies also showed that selective pressures resulting from overuse of antibiotics in agricultural production can lead to the presence of drug resistance(39)(40). And the warning from researchers were that long-term use of antibiotics can lead to the persistence of resistance genes in the gut(41). Therefore, the problem of antibiotic resistance brought by antibiotic selective pressure due to high-density feeding pattern should not be ignored.

Further, the predominant shared ARGs types were tetracycline, quinolone and $\beta$-lactam resistance genes in group of beef and dairy cattle via metagenomic approaches in the test, which were also found in other species of animals, humans or soils(16)(42)(43)(44)(45)(46). And tetracycline and quinolone resistance genes in beef and dairy cattle were obviously higher than those in yak group. The high percentage of tetracycline and quinolone resistance genes might be caused not only by antibiotic selection pressure, but also by horizontal transmission due to high-density feeding pattern. More and more evidences showed that the MGEs was an important way in the mechanism of resistance to tetracycline and quinolones(47) (48)(49)(50). On the other hand, there was a correlation between these ARGs that might lead to multidrug resistance. For example, quinolone resistance genes existing in multiresistance plasmids were linked with other ARGs, like $\beta$-lactamase genes(51).

Recently, many studies showed that MGEs carrying genes of varying activity may lead to selection for resistance, indicating that MGEs was deemed as an important element in the prevalence of ARGs(52) (53). Yaks were seldom exposed to antibiotics but still harbor resistant genes in their intestinal tract, which might be caused by MGEs for delivering ARGs. MGEs consists of insertion sequence, integron, transposons, plasmids, genomic islands and so on. As an important element of MGEs involved in the development of resistance, integron can capture and integrate exogenous genes and spread ARGs horizontally in bacteria through transposons or plasmids via site-specific recombination (52)(54)(55)(56). In our study, it's notable that the abundance of integron in yak gut was higher than that in other two groups, suggesting that high abundance of MGEs in yak may have much stronger ability to transfer ARGs and potential to spread ARGs than that in beef and dairy cattle. And it partly explains why the intestinal microbiome of yaks are rarely exposed to antibiotics but carrying the ARGs. And it's reported that integron mediated ARGs can be transferred from one strain to another derived from the bovine feces and storm water(57). More seriously, more than 80 gene cassettes of class one integrons can become resistant to all $\beta$-lactam and all aminoglycosides(58)(59). Thus, capture systems of integrons allow bacteria to adapt to the challenges of antibiotic treatment regime. This integron-mediated interspecific transfer of ARGs is a great threat to antibiotic therapy in clinic use. And what the importance of this capture system is that it's not just about the phenomenon drug resistance(59). 
Overall, in this study, most data showed that the difference among groups of yak, beef and dairy cattle relating on predominant ARGs types was striking, and the abundance and diversity of ARGs in yak was lower than those in beef and dairy cattle. It implied that yaks exposed to less antibiotics may be less likely to develop resistance than beef dairy cattle and cows because of the antibiotic selective pressure due to different feeding patterns. However, two points are worth noting: first, the group of Yaks are seldom exposed to antibiotics but still harbor a certain amount of ARGs in their intestinal tract; second, high levels of integron were found in the intestinal tract of yaks. These conditions suggest that ARGs may be transmitted horizontally across species via integron-mediated transmission.

\section{Declarations}

\section{Ethics approval and consent to participate}

All animal studies were performed according to the US National Institutes of Health Guidelines for the Care and Use of Laboratory Animals, and approved by Institutional Animal Care and Use Committee of Lanzhou Institute of Husbandry and Pharmaceutical Science of CAAS.

\section{Consent for publication}

Not applicable.

\section{Data availability statement}

The data used to support the findings of this study are included within the article and supplementary information files.

\section{Conflict of interests}

The authors declare that they have no conflict of interests.

\section{Funding}

This work was supported in part by the research on the National Natural Science Foundation of China (grant number 3187520) and Drug Development and Clinical Drug Use Posts of National Beef Yak Industry Technical System [grant number CARS-37].

\section{Authors' contributions}

JY and WW conceived and design this study. WW, LY and FC performed the experiment. BL and XZ analyzed the data. WW and $X J$ interpret the reference and provided the information about the background. WW, XF and JY wrote the paper.

\section{Acknowledgements}


The authors appreciate the Zhang Lu for her generous report on the background information. And the authors are also grateful for the anonymous reviewers for their insightful suggestions and comments.

\section{References}

1. Liu K, Han J, Li S, Liu L, Lin W, Luo J. 2019. Insight into the diversity of antibiotic resistance genes in the intestinal bacteria of shrimp Penaeus vannamei by culture-dependent and independent approaches. Ecotoxicology and Environmental Safety.

2. Aarestrup F. 2012. Sustainable farming: Get pigs off antibiotics. Nature.

3. FDA. 2018. Summary Report on Antimicrobials Sold or Distributed for Use in Food-Producing Animals. Center for Veterinary Medicine.

4. Hu Y, Cheng H. 2014. Research opportunities for antimicrobial resistance control in chinas factory farming. Environmental Science and Technology.

5. McEachran AD, Blackwell BR, Hanson JD, Wooten KJ, Mayer GD, Cox SB, Smith PN. 2015. Antibiotics, bacteria, and antibiotic resistance genes: Aerial transport from cattle feed yards via particulate matter. Environmental Health Perspectives.

6. Allen HK. 2014. Antibiotic resistance gene discovery in food-producing animals. Current Opinion in Microbiology.

7. Van Boeckel TP, Brower C, Gilbert M, Grenfell BT, Levin SA, Robinson TP, Teillant A, Laxminarayan R. 2015. Global trends in antimicrobial use in food animals. Proceedings of the National Academy of Sciences of the United States of America.

8. Carson CA, Reid-Smith R, Irwin RJ, Martin WS, McEwen SA. 2008. Antimicrobial use on 24 beef farms in Ontario. Canadian Journal of Veterinary Research.

9. Lhermie G, Dupouy V, El Garch F, Ravinet N, Toutain PL, Bousquet-Mélou A, Seegers H, Assié S. 2017. Impact of Low and High Doses of Marbofloxacin on the Selection of Resistant Enterobacteriaceae in the Commensal Gut Flora of Young Cattle: Discussion of Data from 2 Study Populations. Foodborne Pathogens and Disease.

10. Pal C, Bengtsson-Palme J, Kristiansson E, Larsson DGJ. 2016. The structure and diversity of human, animal and environmental resistomes. Microbiome.

11. Johnson TA, Stedtfeld RD, Wang Q, Cole JR, Hashsham SA, Looft T, Zhu YG, Tiedje JM. 2016. Clusters of antibiotic resistance genes enriched together stay together in swine agriculture. mBio.

12. Zhou B, Wang C, Zhao Q, Wang Y, Huo M, Wang J, Wang S. 2016. Prevalence and dissemination of antibiotic resistance genes and coselection of heavy metals in Chinese dairy farms. Journal of Hazardous Materials.

13. Tian Z, Zhang Y, Yu B, Yang M. 2016. Changes of resistome, mobilome and potential hosts of antibiotic resistance genes during the transformation of anaerobic digestion from mesophilic to thermophilic. Water Research. 
14. Smillie CS, Smith MB, Friedman J, Cordero OX, David LA, Alm EJ. 2011. Ecology drives a global network of gene exchange connecting the human microbiome. Nature.

15. Chagas TPG, Seki LM, Cury JC, Oliveira JAL, Dávila AMR, Silva DM, Asensi MD. 2011.

Multiresistance, beta-lactamase-encoding genes and bacterial diversity in hospital wastewater in Rio de Janeiro, Brazil. Journal of Applied Microbiology.

16. Xiong W, Wang Y, Sun Y, Ma L, Zeng Q, Jiang X, Li A, Zeng Z, Zhang T. 2018. Antibiotic-mediated changes in the fecal microbiome of broiler chickens define the incidence of antibiotic resistance genes. Microbiome.

17. Li B, Yang Y, Ma L, Ju F, Guo F, Tiedje JM, Zhang T. 2015. Metagenomic and network analysis reveal wide distribution and co-occurrence of environmental antibiotic resistance genes. ISME Journal.

18. Luo R, Liu B, Xie Y, Li Z, Huang W, Yuan J, He G, Chen Y, Pan Q, Liu Y, Tang J, Wu G, Zhang H, Shi Y, Liu Y, Yu C, Wang B, Lu Y, Han C, Cheung DW, Yiu SM, Peng S, Xiaoqian Z, Liu G, Liao X, Li Y, Yang H, Wang J, Lam TW, Wang J. 2012. SOAPdenovo2: An empirically improved memory-efficient short-read de novo assembler. GigaScience.

19. Qin N, Yang F, Li A, Prifti E, Chen Y, Shao L, Guo J, Le Chatelier E, Yao J, Wu L, Zhou J, Ni S, Liu L, Pons N, Batto JM, Kennedy SP, Leonard P, Yuan C, Ding W, Chen Y, Hu X, Zheng B, Qian G, Xu W, Ehrlich SD, Zheng S, Li L. 2014. Alterations of the human gut microbiome in liver cirrhosis. Nature.

20. Nielsen HB, Almeida M, Juncker AS, Rasmussen S, Li J, Sunagawa S, Plichta DR, Gautier L, Pedersen AG, Le Chatelier E, Pelletier E, Bonde I, Nielsen T, Manichanh C, Arumugam M, Batto JM, Quintanilha Dos Santos MB, Blom N, Borruel N, Burgdorf KS, Boumezbeur F, Casellas F, Doré J, Dworzynski P, Guarner F, Hansen T, Hildebrand F, Kaas RS, Kennedy S, Kristiansen K, Kultima JR, Léonard P, Levenez F, Lund O, Moumen B, Le Paslier D, Pons N, Pedersen O, Prifti E, Qin J, Raes J, Sørensen S, Tap J, Tims S, Ussery DW, Yamada T, Renault P, Sicheritz-Ponten T, Bork P, Wang J, Brunak S, Ehrlich SD. 2014. Identification and assembly of genomes and genetic elements in complex metagenomic samples without using reference genomes. Nature Biotechnology.

21. Sunagawa S, Coelho LP, Chaffron S, Kultima JR, Labadie K, Salazar G, Djahanschiri B, Zeller G, Mende DR, Alberti A, Cornejo-Castillo FM, Costea PI, Cruaud C, D’Ovidio F, Engelen S, Ferrera I, Gasol JM, Guidi L, Hildebrand F, Kokoszka F, Lepoivre C, Lima-Mendez G, Poulain J, Poulos BT, Royo-Llonch M, Sarmento H, Vieira-Silva S, Dimier C, Picheral M, Searson S, Kandels-Lewis S, Boss E, Follows M, Karp-Boss L, Krzic U, Reynaud EG, Sardet C, Sieracki M, Velayoudon D, Bowler C, De Vargas C, Gorsky G, Grimsley N, Hingamp P, ludicone D, Jaillon O, Not F, Ogata H, Pesant S, Speich S, Stemmann L, Sullivan MB, Weissenbach J, Wincker P, Karsenti E, Raes J, Acinas SG, Bork P. 2015. Structure and function of the global ocean microbiome. Science.

22. Li J, Wang J, Jia H, Cai X, Zhong H, Feng Q, Sunagawa S, Arumugam M, Kultima JR, Prifti E, Nielsen T, Juncker AS, Manichanh C, Chen B, Zhang W, Levenez F, Wang J, Xu X, Xiao L, Liang S, Zhang D, Zhang Z, Chen W, Zhao H, Al-Aama JY, Edris S, Yang H, Wang J, Hansen T, Nielsen HB, Brunak S, Kristiansen K, Guarner F, Pedersen O, Doré J, Ehrlich SD, Bork P. 2014. An integrated catalog of reference genes in the human gut microbiome. Nature Biotechnology. 
23. Li W, Godzik A. 2006. Cd-hit: A fast program for clustering and comparing large sets of protein or nucleotide sequences. Bioinformatics.

24. Fu L, Niu B, Zhu Z, Wu S, Li W. 2012. CD-HIT: Accelerated for clustering the next-generation sequencing data. Bioinformatics.

25. Karlsson FH, Fåk F, Nookaew I, Tremaroli V, Fagerberg B, Petranovic D, Bäckhed F, Nielsen J. 2012. Symptomatic atherosclerosis is associated with an altered gut metagenome. Nature Communications.

26. Buchfink B, Xie C, Huson DH. 2014. Fast and sensitive protein alignment using DIAMOND. Nature Methods.

27. Huson DH, Mitra S, Ruscheweyh HJ, Weber N, Schuster SC. 2011. Integrative analysis of environmental sequences using MEGAN4. Genome Research.

28. Martínez JL, Coque TM, Baquero F. 2015. What is a resistance gene? Ranking risk in resistomes. Nature Reviews Microbiology.

29. Jia B, Raphenya AR, Alcock B, Waglechner N, Guo P, Tsang KK, Lago BA, Dave BM, Pereira S, Sharma AN, Doshi S, Courtot M, Lo R, Williams LE, Frye JG, Elsayegh T, Sardar D, Westman EL, Pawlowski AC, Johnson TA, Brinkman FSL, Wright GD, McArthur AG. 2017. CARD 2017: Expansion and modelcentric curation of the comprehensive antibiotic resistance database. Nucleic Acids Research.

30. McArthur AG, Waglechner N, Nizam F, Yan A, Azad MA, Baylay AJ, Bhullar K, Canova MJ, De Pascale G, Ejim L, Kalan L, King AM, Koteva K, Morar M, Mulvey MR, O’Brien JS, Pawlowski AC, Piddock LJV, Spanogiannopoulos P, Sutherland AD, Tang I, Taylor PL, Thaker M, Wang W, Yan M, Yu T, Wright GD. 2013. The comprehensive antibiotic resistance database. Antimicrobial Agents and Chemotherapy.

31. Lok C. 2015. Mining the microbial dark matter. Nature.

32. Xiong W, Sun Y, Zeng Z. 2018. Antimicrobial use and antimicrobial resistance in food animals. Environmental Science and Pollution Research.

33. Teillant A, Laxminarayan R. 2015. Economics of Antibiotic Use in U . S . Swine and Poultry Production. Choices.

34. Aarestrup FM. 2005. Veterinary drug usage and antimicrobial resistance in bacteria of animal origin. Basic and Clinical Pharmacology and Toxicology.

35. Chen M, Sun Y, Yang C, Zeng G, Li Z, Zhang J. 2018. The road to wild yak protection in China. Science.

36. Casey JA, Curriero FC, Cosgrove SE, Nachman KE, Schwartz BS. 2013. High-density livestock operations, crop field application of manure, and risk of community-associated methicillin-resistant Staphylococcus aureus infection in Pennsylvania. JAMA Internal Medicine.

37. Casey JA, Shopsin B, Cosgrove SE, Nachman KE, Curriero FC, Rose HR, Schwartz BS. 2014. Highdensity livestock production and molecularly characterized MRSA infections in Pennsylvania. Environmental Health Perspectives. 
38. Woolhouse M, Ward M, Van Bunnik B, Farrar J. 2015. Antimicrobial resistance in humans, livestock and the wider environment. Philosophical Transactions of the Royal Society B: Biological Sciences.

39. Verraes C, Van Boxstael S, Van Meervenne E, Van Coillie E, Butaye P, Catry B, de Schaetzen MA, Van Huffel X, Imberechts H, Dierick K, Daube G, Saegerman C, De Block J, Dewulf J, Herman L. 2013. Antimicrobial resistance in the food chain: A review. International Journal of Environmental Research and Public Health.

40. Buffie CG, Pamer EG. 2013. Microbiota-mediated colonization resistance against intestinal pathogens. Nature Reviews Immunology.

41. Jernberg C, Löfmark S, Edlund C, Jansson JK. 2010. Long-term impacts of antibiotic exposure on the human intestinal microbiota. Microbiology.

42. Ma L, Xia Y, Li B, Yang Y, Li LG, Tiedje JM, Zhang T. 2016. Metagenomic Assembly Reveals Hosts of Antibiotic Resistance Genes and the Shared Resistome in Pig, Chicken, and Human Feces. Environmental Science and Technology.

43. Taylor NM, Clifton-Hadley FA, Wales AD, Ridley A, Davies RH. 2009. Farm-level risk factors for fluoroquinolone resistance in E. coli and thermophilic Campylobacter spp. on finisher pig farms. Epidemiology and Infection.

44. Barton MD. 2014. Impact of antibiotic use in the swine industry. Current Opinion in Microbiology.

45. Forsberg KJ, Patel S, Gibson MK, Lauber CL, Knight R, Fierer N, Dantas G. 2014. Bacterial phylogeny structures soil resistomes across habitats. Nature.

46. Forsberg KJ, Reyes A, Wang B, Selleck EM, Sommer MOA, Dantas G. 2012. The shared antibiotic resistome of soil bacteria and human pathogens. Science.

47. Mullany P, Allan E, Warburton PJ. 2012. Tetracycline resistance genes and mobile genetic elements from the oral metagenome. Clinical Microbiology and Infection.

48. Petkovic S, Hinrichs W. 2017. Blocking tetracycline destruction. Nature Chemical Biology.

49. Jacoby GA. 2017. Plasmid-Mediated Quinolone ResistanceAntimicrobial Drug Resistance.

50. Li XZ. 2005. Quinolone resistance in bacteria: Emphasis on plasmid-mediated mechanisms. International Journal of Antimicrobial Agents.

51. Jacoby GA, Hooper DC. 2014. Review of the quinolone familyAntibiotic Discovery and Development.

52. Gaze WH, Zhang L, Abdouslam NA, Hawkey PM, Calvo-Bado L, Royle J, Brown H, Davis S, Kay P, Boxall ABA, Wellington EMH. 2011. Impacts of anthropogenic activity on the ecology of class 1 integrons and integron-associated genes in the environment. ISME Journal.

53. Von Wintersdorff CJH, Penders J, Van Niekerk JM, Mills ND, Majumder S, Van Alphen LB, Savelkoul PHM, Wolffs PFG. 2016. Dissemination of antimicrobial resistance in microbial ecosystems through horizontal gene transfer. Frontiers in Microbiology.

54. Hall RM, Stokes HW. 1993. Integrons: Novel DNA elements which capture genes by site-specific recombination. Genetica. 
55. Collis CM, Grammaticopoulos G, Briton J, Stokes HW, Hall RM. 1993. Site-specific insertion of gene cassettes into integrons. Molecular Microbiology.

56. Hall RM, Collis CM. 1995. Mobile gene cassettes and integrons: capture and spread of genes by sitespecific recombination. Molecular Microbiology.

57. Nagachinta S, Chen J. 2008. Transfer of class 1 integron-mediated antibiotic resistance genes from shiga toxin-producing Eschenchia coli to a susceptible E. coli K-12 strain in storm water and bovine feces. Applied and Environmental Microbiology.

58. Rowe-Magnus DA, Mazel D. 2002. The role of integrons in antibiotic resistance gene capture. International Journal of Medical Microbiology.

59. Mazel D. 2006. Integrons: Agents of bacterial evolution. Nature Reviews Microbiology.

\section{Figures}
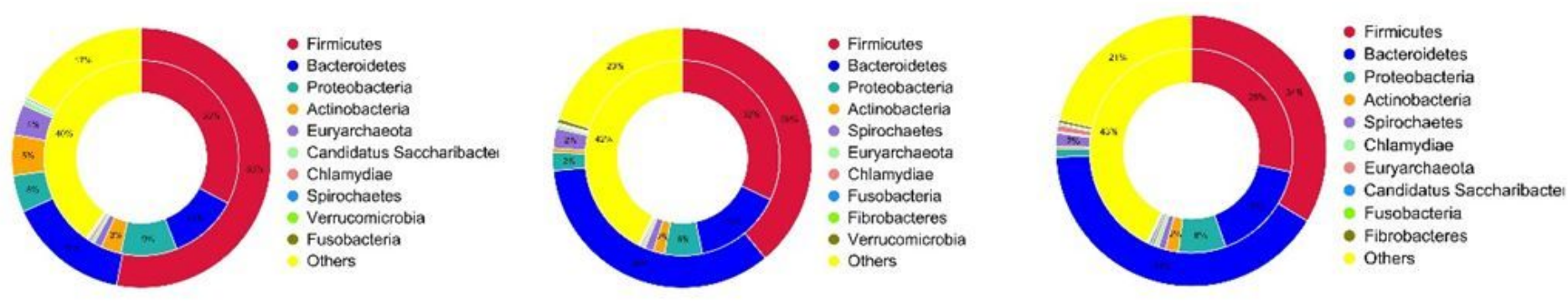

\section{Figure 1}

Comparison of the distribution of the ARGs and the bacterial gene sets at the phylum level of yak, beef and dairy cattle, respectively. The inner circle is the species distribution of ARG, while the outer circle is the species distribution of all sample genes in the group. 


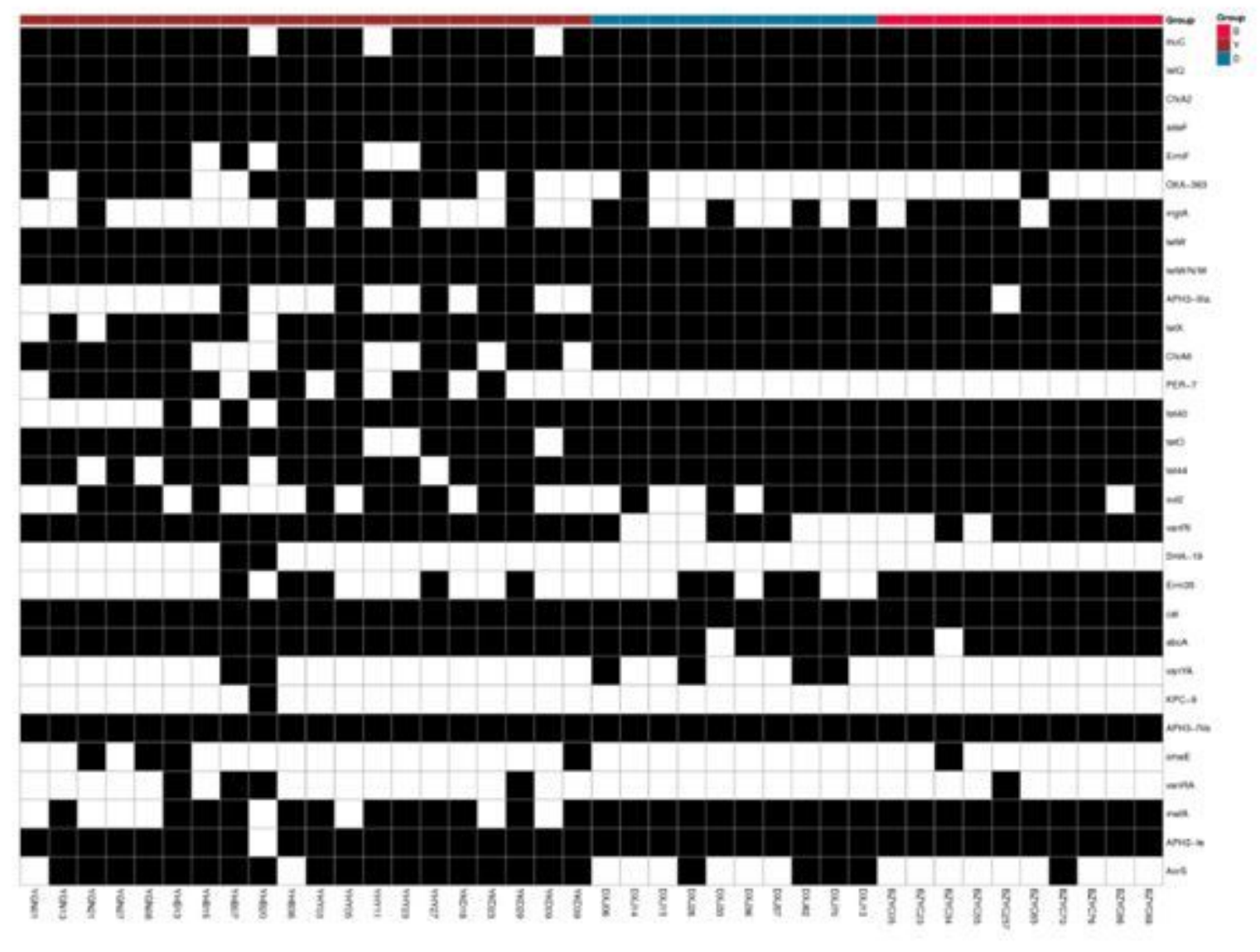

Figure 2

The heat map of top30 ARG distribution, the horizontal axis is the sample name, and the right vertical axis is the ARG name of resistance gene type. Black represents the ARO in the sample, while white represents the ARG in the sample 


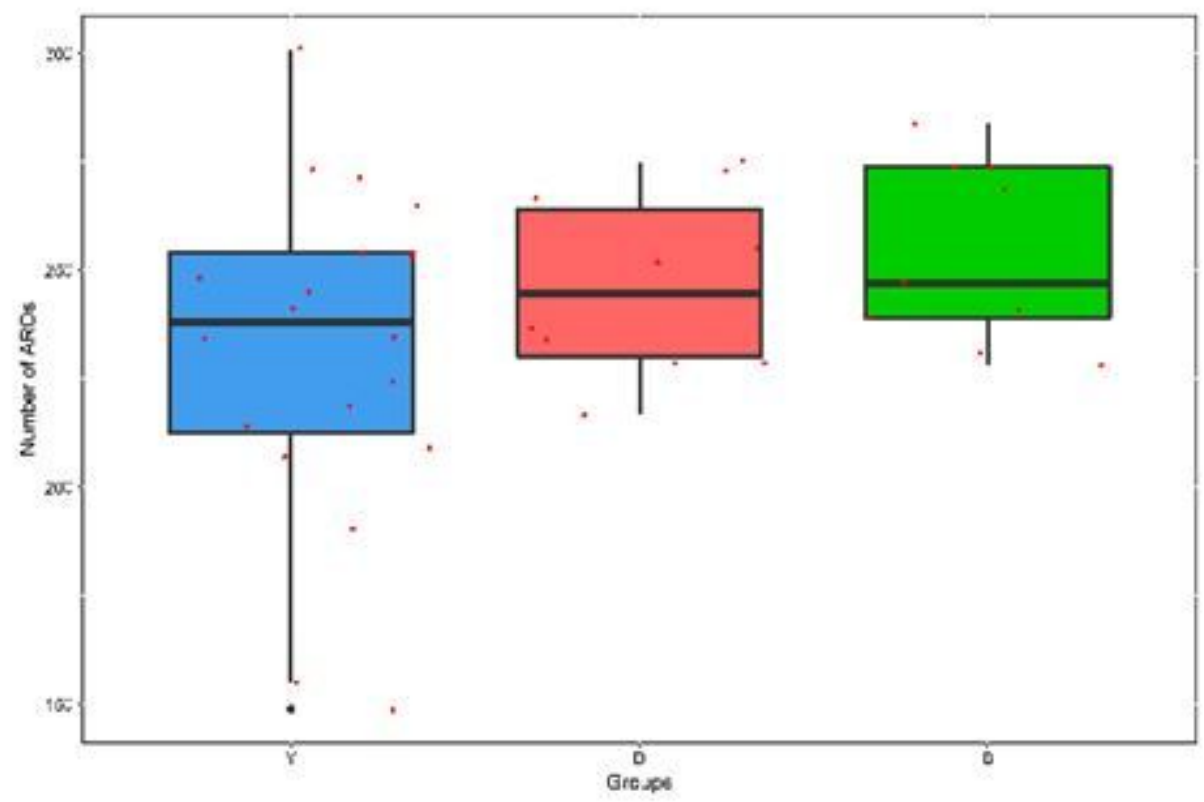

Figure 3

The difference on the number of ARGs among yak, beef and dairy cattle is shown in the boxpot.

a

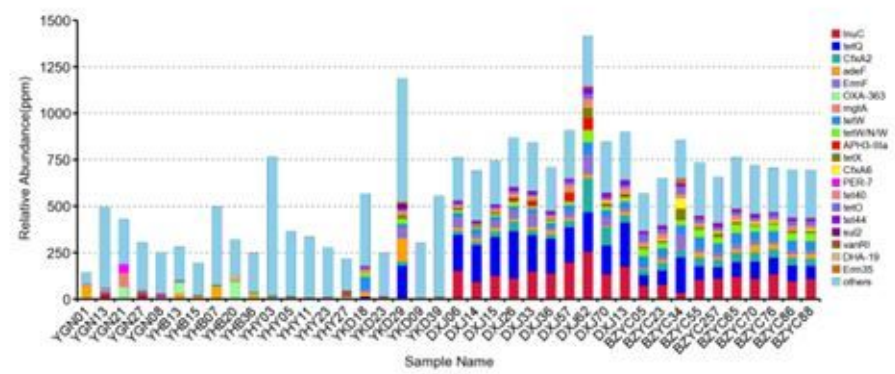

b

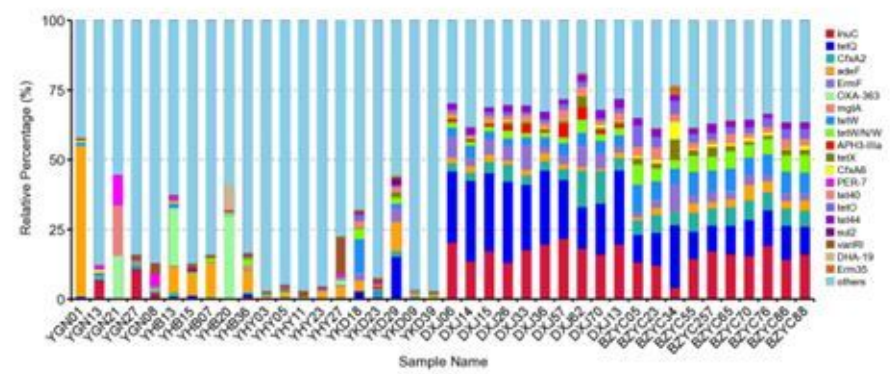

Figure 4

(a) represents the relative abundance of ARGs in each sample, and the unit PPM is the result of amplifying the original relative abundance data by 106 times; (b) represents the relative abundance of top20 ARGs in all ARGs, and others represent the total relative abundance of non-top 20 ARGs. 


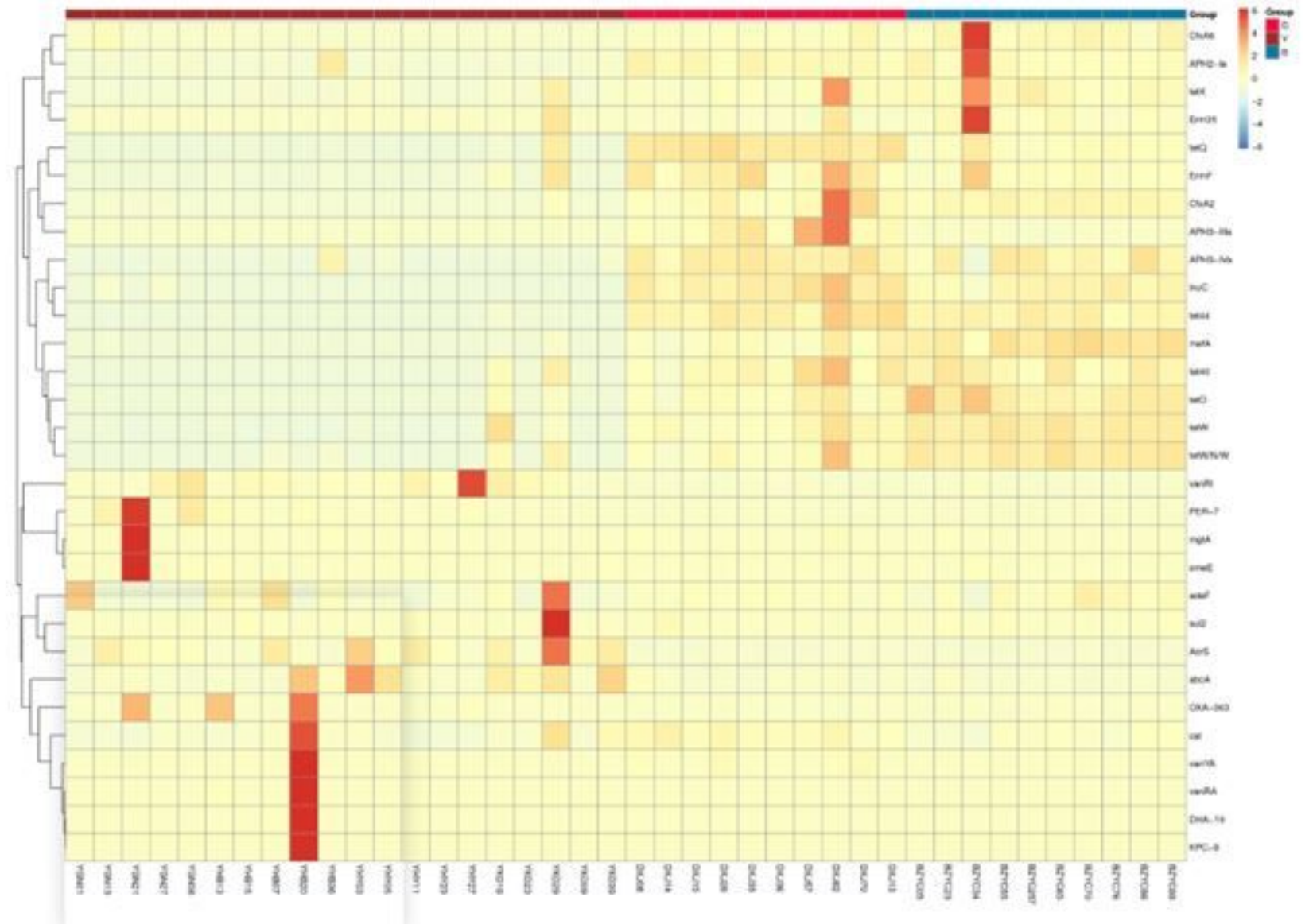

Figure 5

The top 30 ARG abundance clustering heat map. The right vertical axis is the ARG name, the left vertical axis is the ARG clustering tree, and the corresponding value of the intermediate heat map is the $Z$ value of ARG relative abundance in each row after standardized processing. 


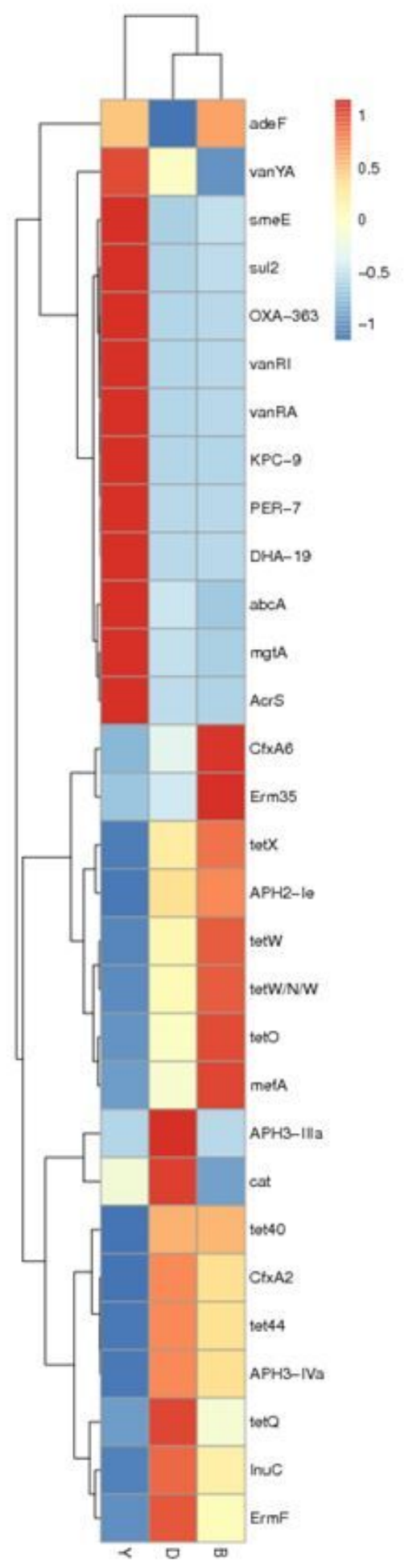

Figure 6

Heatmap variations of the relative abundance level of each top30 ARGs subtype among the group of yak, beef and dairy cattle. The right vertical axis is the name of ARG subtype, and the bottom of horizontal axis is the group name. The left vertical axis is cluster tree. 


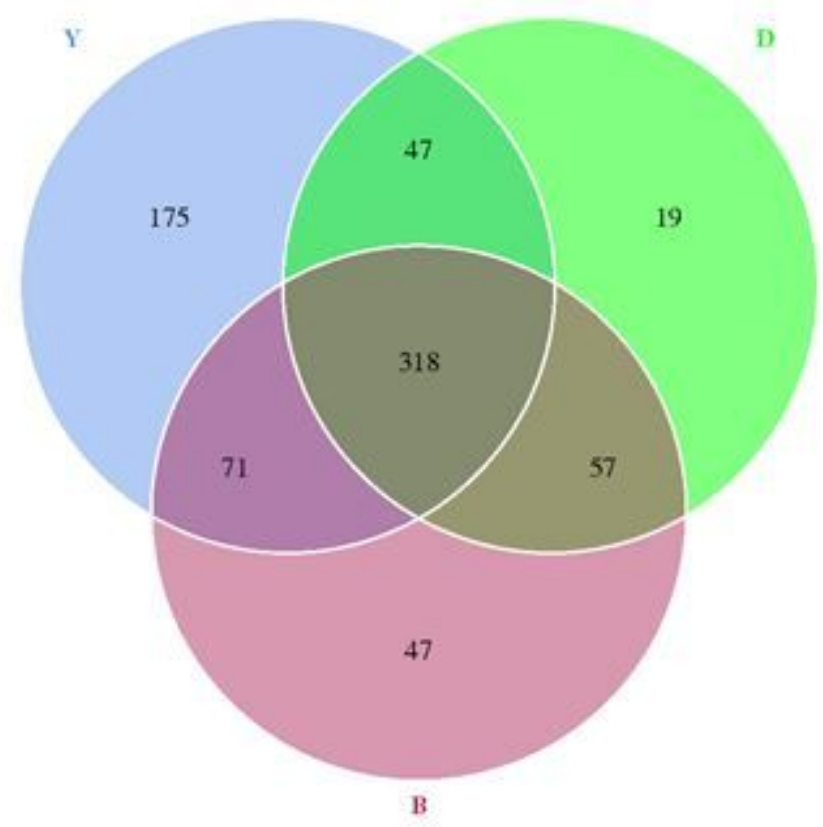

Figure 7

The Venn diagram showing the number of shared ARGs in yak gut, beef gut and dairy cattle gut

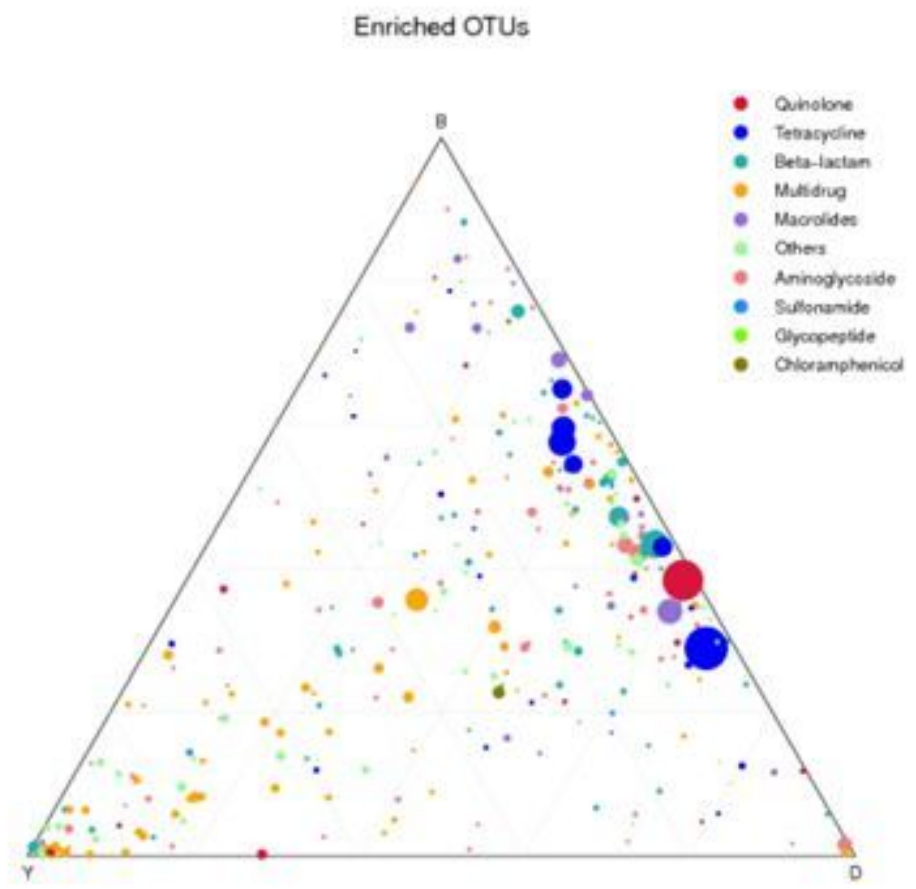

Figure 8 
Ternary plot showing the abundance comparison of 318 shared ARGs in yak gut, beef gut and dairy cattle gut. The sum of the abundance for one species ARG in these three types of gut was set as $100 \%$. The percentage of each certain ARG in each gut is equal to its corresponding abundance which is divided by the abundance sum of this ARG in the three groups of gut.

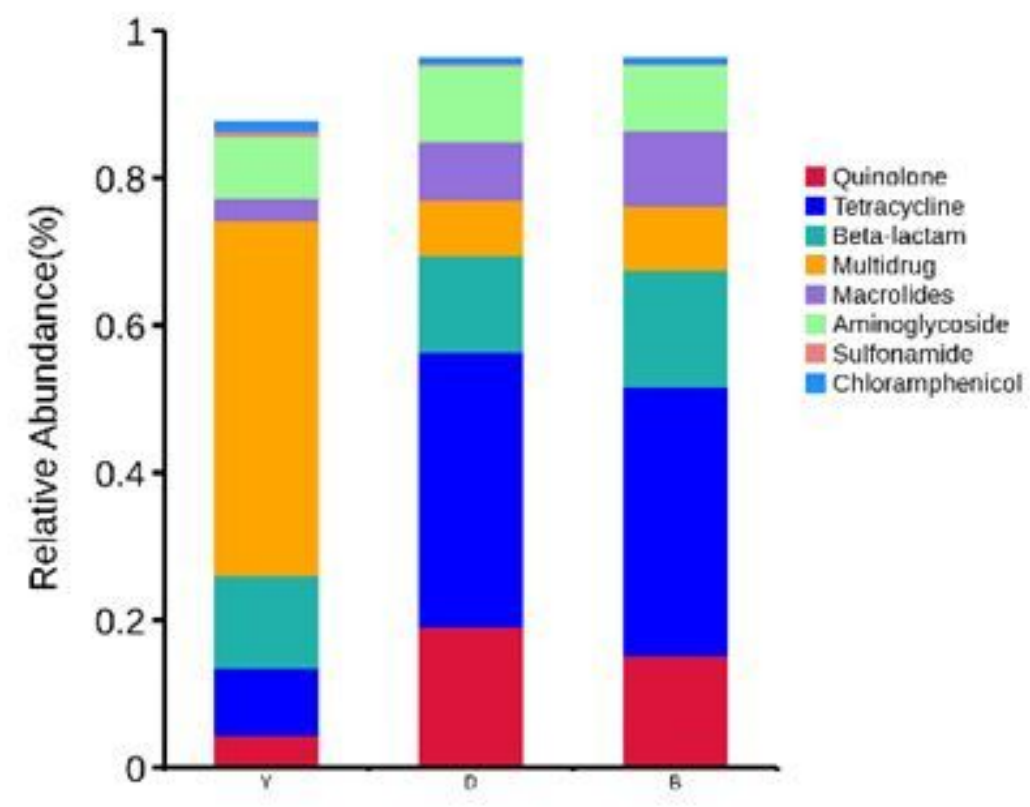

Figure 9

The relative abundance of shared ARGs types assigned to each major antibiotic class among the group of yak, beef and dairy cattle.
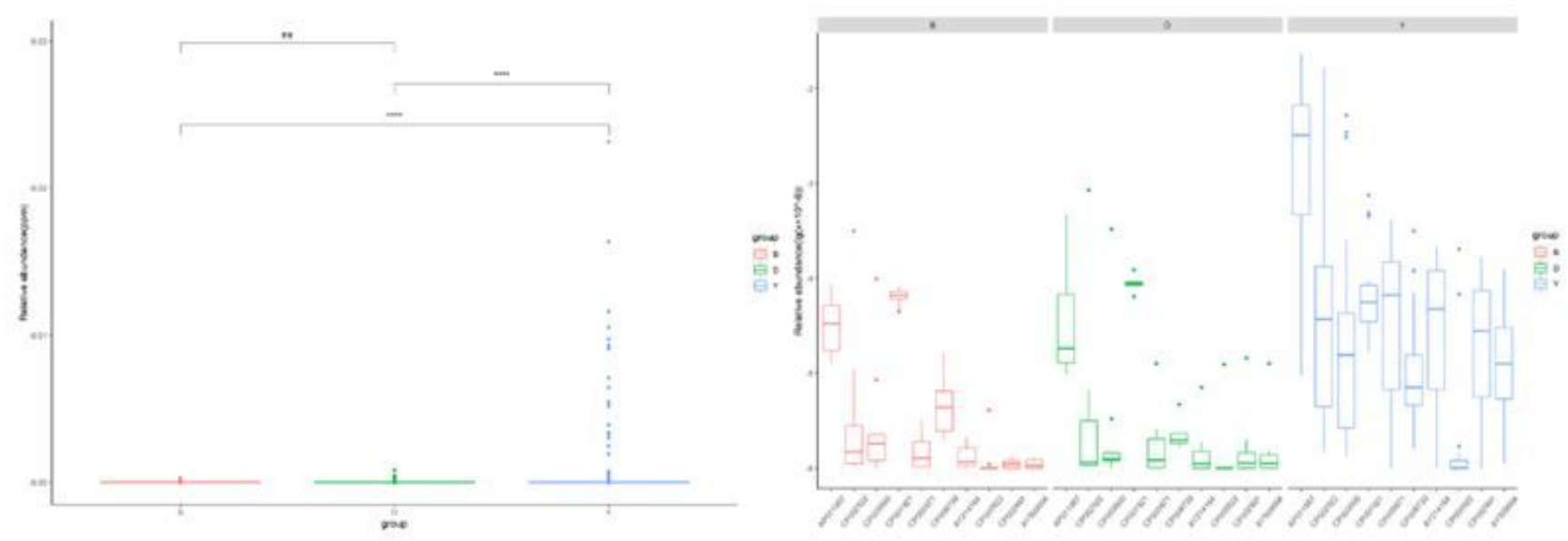

Figure 10 
(a)The difference of relative abundance of mobile genetic elements (integron) among yak, beef and dairy cattle is shown in the sigbox. ( ${ }^{\star}$ represents $p<0.005, \star \star \star$ represents $p<0.001$, $* \star \star \star$ represents $p<0.0001$ )

(b)The difference of the abundance of top ten mobile genetic elements (integron) in the yak, beef and dairy cattle.

\section{Supplementary Files}

This is a list of supplementary files associated with this preprint. Click to download.

- Supplementrytables1tos4.docx 